\title{
Geschichte der Hautklinik in Gera, von der Gründung bis Dezember 2010 und aktueller Ausblick
}

\author{
History of the Dermatological Department in Gera, Past, Present and Future
}

Autoren

Institut
M. Kaatz, J. Meyer

Klinik für Hautkrankheiten und Allergologie am SRH Waldklinikum Gera GmbH
Bibliografie

DOI http://dx.doi.org/

$10.1055 / \mathrm{s}-0032-1326481$

Akt Dermatol 2013; 39: 115-118

(c) Georg Thieme Verlag KG

Stuttgart · New York

ISSN 0340-2541

\section{Korrespondenzadresse}

\section{PD Dr. Martin Kaatz}

Klinik für Hautkrankheiten und Allergologie am SRH Waldklinikum Gera GmbH Str. des Friedens 122

07548 Gera

Martin.kaatz@wkg.srh.de

\section{Zusammenfassung \\ $\nabla$}

Die Hautklinik in Gera hat eine nunmehr über 60 Jahre währende Tradition und ist ein wesentlicher Bestandteil in der stationären Versorgung von dermatologischen Patienten in Ostthüringen. In ihrer gesamten Geschichte wurden frühzeitig innovative Therapieverfahren in die Behandlung von Dermatosen und Hauttumoren eingeführt. Geprägt wurde die Klinik vor allem durch Herrn

\section{Bis 1945}

\section{$\nabla$}

Bereits in den Jahren 1928 (169 dermatologische Patienten) und 1929 (166 dermatologische Patienten) zeigte sich auf den Inneren Abteilungen (I - Frauen, II - Männer) des kürzlich gegründeten Waldkrankenhauses in Gera ein hoher Bedarf an dermato-venerologischen Leistungen, die zum damaligen Zeitpunkt vom zuständigen Stadtarzt in Gera erbracht wurden. Damit wuchs der Wunsch nach einer eigenen Fachabteilung. Am 15. Oktober 1935 erfolgte die Eröffnung einer ersten Fachstation für Dermatovenerologie im Städtischen Waldkrankenhaus (noch der Inneren Medizin angegliedert), die von Herrn Dr. med. Gisbert Wesener geleitet wurde. Dr. Wesener hatte 1932 in Würzburg promoviert und danach in Breslau gearbeitet.

\section{Nach 1945}

\section{$\nabla$}

Nach dem 2.Weltkrieg waren Hautkranke in einem Gebäude (Loreystr. 6) mit schlechten sanitären und hygienischen Verhältnissen, das zunächst dem Notlazarett bzw. „Behelfskrankenhaus Schillerschule“ angeschlossen war, untergebracht. Bei steigenden dermatologischen Patientenzahlen wurde 1949 ein Investitionsantrag gestellt, der 1950 vom Thüringer Ministerium für
ChA Meyer, der sie über 30 Jahre geleitet hat. Aktuell verfügt das Haus über 30 vollstationäre und 5 tagesstationäre Betten, einen modernen Funktionsbereich mit den Schwerpunkten Lichtabteilung und Allergologie. Gleichzeitig wurden die operativen Angebote in den letzten Jahren als Bestandteil der Dermato-Onkologie deutlich ausgebaut. Im nächsten Jahr wird die Klinik ihr neues Domizil in modernen Räumlichkeiten beziehen.

Arbeit und Sozialwesen in Erfurt genehmigt wurde. Im April 1952 wurde die Hautklinik als Klinik der Städtischen Krankenanstalten Gera unter der Leitung von Herrn Chefarzt Dr. Wesener eröffnet. Die Klinik war zu diesem Zeitpunkt auf 130 Betten ausgelegt und mit einer angegliederten Ambulanz auf die Versorgung von Ostthüringen ausgerichtet.

Durch die Gebäudeerweiterung und Sanierung verfügte die Klinik nach Beendigung des 2 . Bauabschnittes über umfangreiche diagnostische und therapeutische Möglichkeiten, darunter eine Bäderabteilung zur Hydrotherapie. Gleichzeitig konnten nach Installation eines Röntgentherapiegerätes Hauttumoren strahlentherapeutisch behandelt werden. Weiterhin bestanden die Voraussetzungen für dermatochirurgische Eingriffe. Mehrere Publikationen der Doctores Wesener, Bock und Frank aus den 50er-Jahren belegen die Einführung moderner Therapieverfahren in Gera, so den Einsatz von Antibiotika, Tuberkulostatika, von ACTH und weiterer Kortikoide. Spezialgebiet des Chefarztes Dr. Wesener war jedoch die Behandlung von „Beingeschwüren durch Venenleiden“. Nach seiner „Übersiedelung“ 1958 nach Aachen war er einer der Mitbegründer der Deutschen Gesellschaft für Phlebologie und erhielt dafür später das Bundesverdienstkreuz. Von seiner herausragenden, über Deutschland hinausgehenden Bedeutung zeugen zahlreiche internationale Publikationen, die sein Wirken würdigen. 


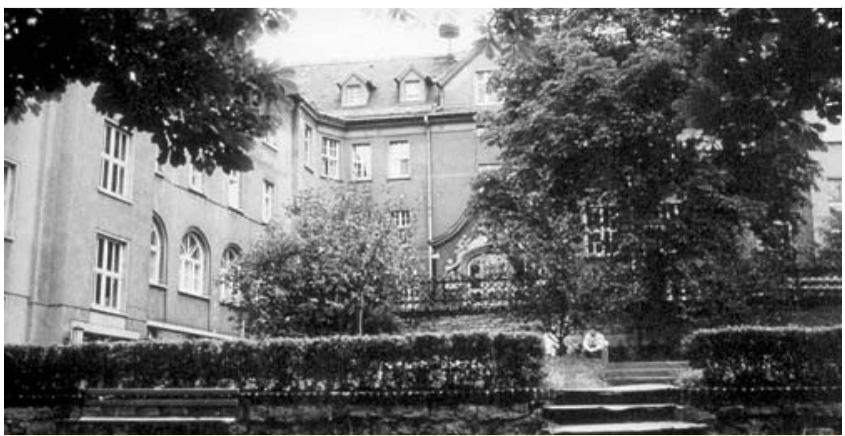

Abb.1 Das Gebäude der Hautklinik in den 70er-Jahren in der Loreystraße.

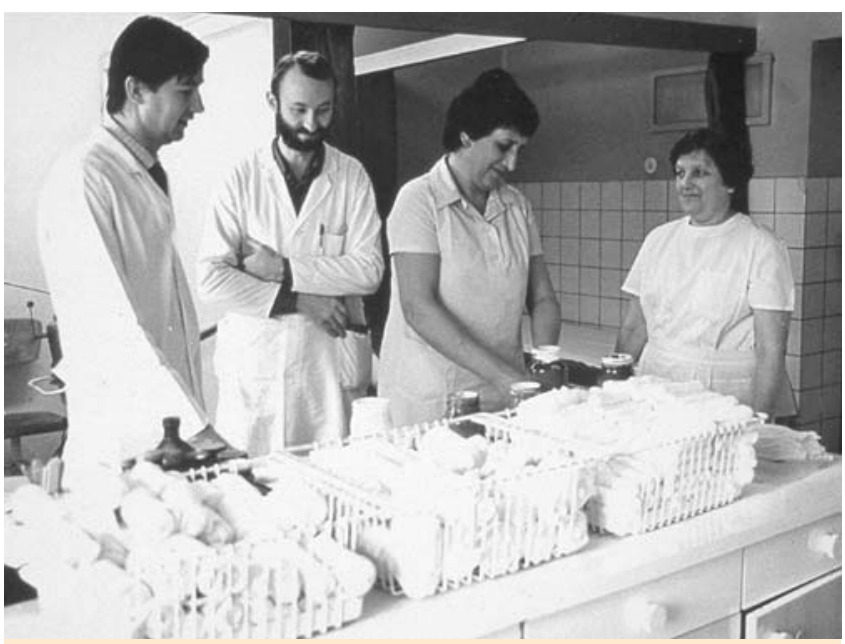

Abb. 2 Chefarzt Meyer im Kreise von Kollegen und Mitarbeiterinnen bei der Vorbereitung der Dermatotherapie.

In den Jahren 1958-1960 verließen vier weitere Fachärzte die Klinik in Richtung Bundesrepublik, sodass Frau Dr. Magdalena Schmidt, die spätere langjährige Oberärztin, die kommissarische Leitung übernahm und die Klinik über eine personell schwierige Zeit brachte.

Am 1.7.1959 übernahm der kommissarische Direktor der Universitäts-Hautklinik Rostock, Prof. Dr. Frank Fleck, die Leitung der Klinik. Seine Frau, Prof. Dr. Margarete Fleck, zuvor Oberärztin an der Hautklinik der Charité Berlin, arbeitete zudem als Oberärztin an der Klinik und war gleichzeitig Leiterin des verwaltungstechnisch getrennten Kreisambulatoriums für Geschlechtskrankheiten. Die Klinik wurde in dieser Zeit neu organisiert und verfügte über insgesamt 93 Betten in drei getrennten Stationen (Frauen- mit angeschlossener venerologischer Abteilung, Männer- und Kinderstation) ( Abb. 1). Während dieser Zeit änderte sich das Profil der Abteilung mit einem Schwerpunkt für die Behandlung von Ekzemen, Psoriasis- und Gefäßerkrankungen sowie den infektiösen Hauterkrankungen. Zudem wurde eine andrologische Diagnostik etabliert und eine der ersten Ehe- und Sexualberatungsstellen in der DDR (1.8.1966) eingeführt. Zuvor war bereits mit dem Epikutantest (1964) erstmalig ein diagnostisches Verfahren zur Untersuchung von Allergien angewendet worden. Auch eine dermatokosmetische Beratung und Behandlung wurde bereits in den 60er-Jahren angeboten. Als Ausdruck der wachsenden Möglichkeiten im Gesundheitswesen ist auch die Angliederung der dermatologischen Kurenleitstelle 1963 zu sehen.

Als besonderen Verdienst der Ära Fleck an der Hautklinik in Gera ist die enge Verzahnung zwischen ambulanter und stationärer
Betreuung zu sehen. So führte Frau Prof. Fleck zweimal wöchentlich eine stationäre Visite durch und entschied über eine weitere ambulante Therapie. Unter Leitung der bereits erwähnten Oberärztin, Frau Dr. Magdalena Schmidt, wurde die Ambulanz der Klinik ausgebaut, was die jährlichen Konsultationszahlen von 21000 bis 32000 unterstreichen. Herr OMR Prof. Dr. F. Fleck und Frau Prof Dr. M. Fleck haben in ihrer aktiven Zeit zahlreiche wissenschaftliche Arbeiten verfasst. Das „Lehrbuch für Haut- und Geschlechtskrankheiten“, eines von drei publizierten Fachbüchern, entwickelte sich zum Standardwerk des Fachgebietes in der damaligen DDR.

Nach seinem altersbedingten Ausscheiden stand die Klinik zwischen dem 1.1.1975-31.12.1975 unter der kommissarischen Leitung von Frau OÄ Dr. M. Schmidt.

Am 1.1.1976 übernahm Dr. Frank Liebsch, vorher Oberarzt der Hautklinik der Karl-Marx-Universität Leipzig, die Leitung der Klinik. Durch zahlreiche Umstrukturierungen mit der Schaffung von Funktionsräumen, Arztzimmern, einer Patientenbibliothek und eines Chefarztsekretariates konnten die Arbeitsbedingungen deutlich verbessert werden. Ein Kabinensystem in den Behandlungszimmern trug zudem dem gewachsenen Bedürfnis nach individueller Betreuung und Schutz der Privatsphäre der Patienten Rechnung. Der Aufbau einer gemischten Frauen- und Kinderstation ermöglichte zudem eine gemeinsame Betreuung von Müttern und Kindern. Die ambulante Versorgung wurde zunehmend in die Polikliniken der Stadt verlagert, bei gleichzeitigem Ausbau stationärer Leistungen insbesondere im Laborbereich. Besonderer Schwerpunkt von Herrn ChA Dr. Liebsch stellte die Betreuung kinderloser Ehepaare und von Patienten mit Sexualerkrankungen dar. Nach schwerer Krankheit verstarb Chefarzt Dr. F. Liebsch am 1.12.1978.

Unter schwierigen Bedingungen wurde die stationäre Versorgung in den folgenden Monaten von Frau OÄ Felgenträger und der Leiterin des Kreisambulatoriums für Geschlechtskrankheiten der Stadt Gera, Frau MR Dr. M. Schmidt, aufrechterhalten.

Am 1.10.1979 wurde Dr. Jochen Meyer, zuvor Oberarzt der Hautklinik der Friedrich-Schiller-Universität Jena, zum Chefarzt der Einrichtung berufen ( $\bullet$ Abb.2). Unter seiner Leitung wurde das Profil der nunmehr 81 Betten umfassenden Klinik den aktuellen modernen diagnostischen und therapeutischen Entwicklungen angepasst. Die dermatokosmetische Abteilung konnte am 1.3.1980 wieder eröffnet werden, gleichzeitig wurde der Bereich Dermatochirurgie deutlich ausgeweitet und ab 1984 die Kryotherapie eingeführt. Mit dem wachsenden Verständnis für kausale Zusammenhänge wurden Therapiestandards etabliert und neue diagnostische, insbesondere immunologische Verfahren etabliert. Unter Chefarzt Meyer sind die Dispensaire-Sprechstunden Zytostatika, Hauttumoren und Andrologie aufgebaut worden. Gleichzeitig wurde die Kurenleitstelle weitergeführt.

In Zusammenarbeit mit der Hautklinik in Jena wurde 1982 die BCG-Immuntherapie zur Behandlung maligner Melanome eingeführt und die Klinik als dermatologisches Diagnostik- und Therapiezentrum entwickelt. 1984 übernahm die Klinik die arbeitsdermatologische Leitstelle der Arbeitshygieneinspektion des Bezirkes Gera und erarbeitete für den Bezirk verbindliche Untersuchungsstandards für die Tauglichkeit in Nassberufen. Im gleichen Jahr erfolgte die Erweiterung des allergologischen Prüfzentrums und des Testlabors nach neuesten Diagnostikstandards.

Im Jahr 1986 wurde eine Lichtabteilung etabliert, die den aktuellen Entwicklungen in der Photodermatologie Rechnung trug. Unter der Leitung von Frau OÄ Felgenträger wurde diese Abteilung in den 90er-Jahren mit moderner Gerätetechnik durch die 
diagnosebezogene Anwendung selektiver UV-Spektren konsequent weiterentwickelt.

Im Oktober 1989 konnte die Klinik unter intensiver Zusammenarbeit mit der Sektion Physik der Friedrich-Schiller-Universität Jena als 2.Dermatologische Einrichtung der damaligen DDR die Argon-Laser-Therapie mittels ILA 120/Carl Zeiss Jena zur Behandlung gutartiger Hautfehlbildungen einführen ( $\bullet$ Abb.3).

Die bauliche Qualität der Hautklinik insbesondere von Sanitäranlagen und Heizung verschlechterte sich in den 80er-Jahren zusehends. In den Jahren 1991 bis 1992 gelang es jedoch, Fördermittel der Bundesrepublik und des Landes Thüringen zu erhalten, die zu einer Gesamtrekonstruktion des gesamten Innenbereiches genutzt wurden. Während der Baumaßnahmen zogen die Hautpatienten in das sogenannte „Schifferhaus“ der Lungenklinik in Gera-Ernsee um, wo unter beengten Bedingungen die stationäre Therapie weitergeführt wurde. Am 2.9.1992 wurde durch den Bürgermeister und Baudezernenten R. Rauch sowie die Dezernentin Frau E. Krumflek die rekonstruierte Hautklinik des Klinikums der Stadt Gera feierlich zur Nutzung übergeben.

Die Klinik verfügte nunmehr über 45 Betten auf zwei Stationen mit angegliederten Funktionsabteilungen. Für die Patienten waren moderne 2- bis 4-Bettzimmer, großzügige Bäder und Aufenthaltsräume geschaffen worden. Neue Arztzimmer und der Einbau eines OP-Bereiches verbesserten zudem das Angebot an modernen Diagnostik- und Therapieverfahren.

Am 24.10.1992 wurde das 40-jährige Jubiläum der Hautklinik mit einem wissenschaftlichen Symposium - „Licht und Onkologie“ - mit Referenten aus Wien und der Partnerstadt Nürnberg begangen. Zahlreiche weitere Fortbildungsveranstaltungen in Gemeinschaft mit dem Berufsverband Deutscher Dermatologen und im Auftrag der Landesärztekammer Thüringen unterstreichen die Rolle der Klinik als Fortbildungseinrichtung. Gleichzeitig erfüllte die Klinik ihre Verantwortung als Lehrkrankenhaus der Friedrich-Schiller-Universität Jena und hat zahlreiche Studenten im Praktischen Jahr und Ärzte im Praktikum ausgebildet. Die Forschung war im Wesentlichen auf praxisbezogene Arzneimittelentwicklung Phase-2/3-Studien, Melanomforschung sowie Arbeitsdermatologie ausgerichtet. Hiervon zeugen die Mitarbeit in Kommissionen zur Neubewertung der MdE bei Berufskrankheiten oder Deutscher Kontaktallergiegruppe und EpikutantestVerbund dermatologischer Kliniken (ab 2001) durch den Chefarzt Doz. Dr. J. Meyer sowie der ADO durch Frau OA Dr. B. Felgenträger.

Neben den weiteren Schwerpunkten der stationären Betreuung wie Dermatochirurgie sowie maligner Hauterkrankungen mit modernen immunologischen Therapieverfahren und standardisierten Nachsorgeprogrammen auch in Zusammenarbeit mit dem Tumorzentrum des Wald-Klinikums, moderner Lichttherapie (ab August $2001 \mathrm{UVA}_{1}$-Lichttherapie), Lasertherapie mittels Argon- und $\mathrm{CO}_{2}$-Laser, kosmetischer Therapiemethoden wie Botulinumtoxin bei Hyperhidrose und Gesichtsfalten, standardisierten Diagnostikverfahren der Pilzzüchtung für das Gesamtklinikum haben sich Umwelt- und Autoimmunerkrankungen sowie allergische Erkrankungen als Schwerpunkte der täglichen Betreuung etabliert.

Als Zentrum zur Therapie von Insektengiftallergien, Arzneimittel- und Nahrungsmittelüberempfindlichkeiten hat sich die Klinik interdisziplinäre Felder neu erarbeitet. So wurde am 1.12.1999 die Klinik entsprechend des Profils als „Klinik für Hautkrankheiten und Allergologie“ umbenannt. In 32 vollstationären und 3 tagesklinischen Betten mit stark verkürzter Verweildauer von 10,9 Tagen (zu 18,7 im Jahr 1989) sowie Aufnahme-

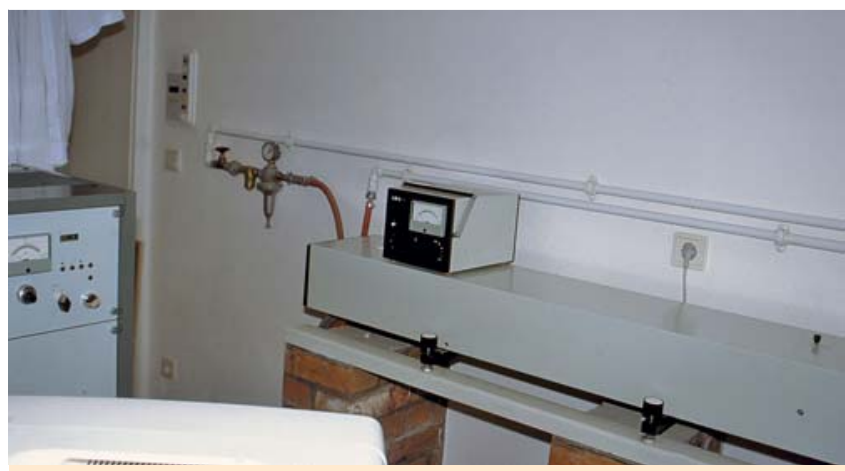

Abb.3 Eine der ersten in der DDR installierten Argonlaseranlagen 1989.

ambulanz und Ambulanz in KV-Spezial- sowie Hospitationssprechstunde hat sich die Klinik den Ruf als Betreuungsschwerpunkt Ostthüringen erhalten.

\section{0plus}

$\nabla$

Im Juli 2005 wurde die Klinik als Außenstandort (Loreystr.) in das Gesamtklinikum integriert. Standort wurde nunmehr die 6. Etage des ehemaligen Wismutkrankenhauses, SRH Klinikum/Standort II, mit 30 vollstätionären und 5 tagesklinischen Betten. Die modern ausgestattete Klinik verfügt am Standort über eine Funktionsabteilung (Laser, OP, Lichtabteilung), ein Labor (Allergologie) und eine Ambulanz. Durch die kurzen Wege hat sich die interdiszipilinäre Zusammenarbeit deutlich verbessert (u.a. radiologische Diagnostik, Konsile, Notfälle). Die Klinik kann zudem auf einen OP für die Allgemeinanästhesie und ein Ultraschallgerät zurückgreifen. Trotz der gesunkenen Bettenzahl konnten die stationären Fallzahlen (920 vollstationär, 200 tagesklinisch) gehalten werden. Gleichzeitig wurden neue Therapieverfahren eingeführt. Im operativen Sektor die Sentinel-Node-Biopsie ab 2003, Erweiterungen in der Lasertherapie, Einführung großer Hautplastikverfahren und die Liposuction unter Federführung der operativen Oberärztinnen Dr. D. Gauß und Dr. A. Durnick. In der Dermatoonkologie erfolgte die Eingliederung der Klinik in das Tumorzentrum mit einer strukturierten Tumornachsorge. Gleichzeitig wurden die Verfahren der Immuntherapie und Chemotherapieverfahren ausgebaut. Im Bereich der Allergologie besteht eine enge Zusammenarbeit auch im Rahmen von Studien mit dem IVDK sowie eine intensive Kooperation mit niedergelassenen Kollegen, die 2006 zur Gründung des Netzwerkes Allergologie führte. Chefarzt PD Meyer erhielt 2009 als langjähriger Vorsitzender und Vorstand des Berufsverbandes Landesgruppe Thüringen als Auszeichnung die „Verdienstmedaille des Berufsverbandes Deutscher Dermatologen“ anlässlich seines 30-jährigen Jubiläums als Chefarzt der Klinik.

\section{Die Klinik für Hautkrankheiten und Allergologie am SRH Waldklinikum Gera - Gegenwart und Ausblick $\nabla$}

Mit dem 31.12.2010 ist Chefarzt Meyer nach über 30 bewegten und überaus erfolgreichen Jahren Berufslebens als Leiter der Hautklinik in den verdienten Unruhestand verabschiedet worden. Die Leitung übernahm Dr. Martin Kaatz vom Universitätsklinikum Jena ( Abb.4). Dabei konnte die Bettenzahl von 30 vollstationären und 6 tagesstationären Betten beibehalten werden. 


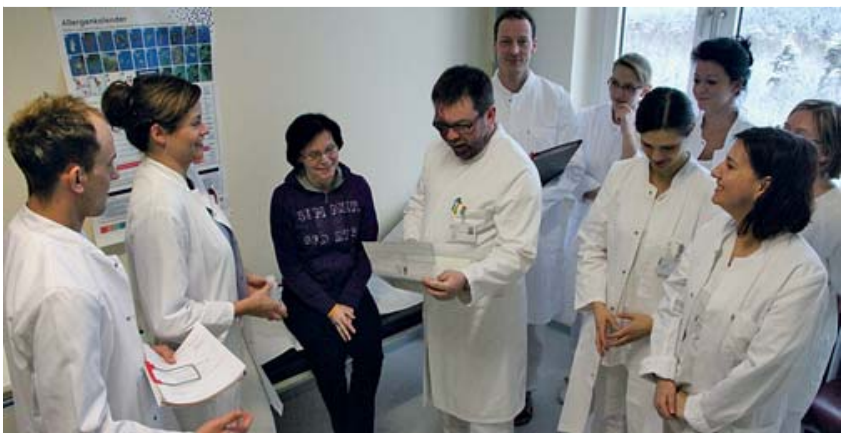

Abb.4 Chefarzt Kaatz bei der Patientenvorstellung.

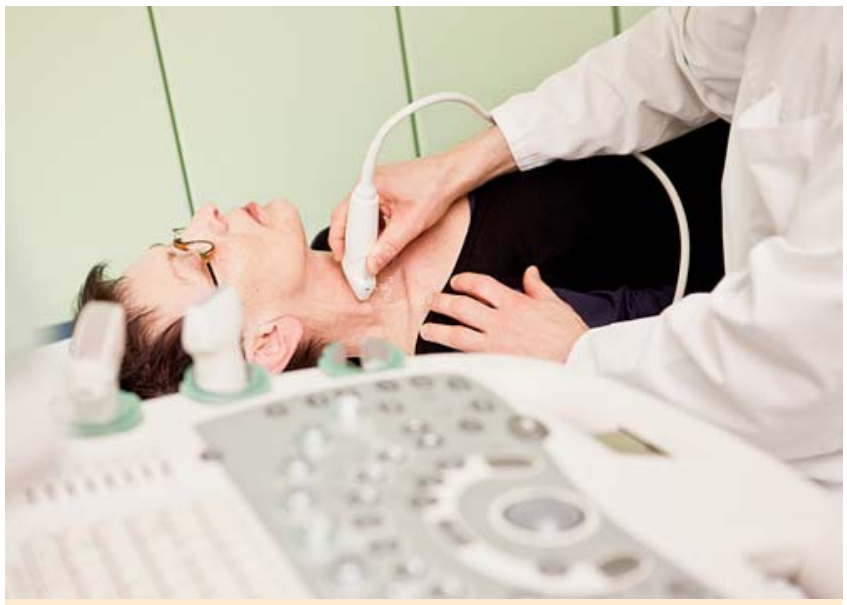

Abb.5 Moderne Ultraschalldiagnostik in der Dermatologie.

Im Jahr 2012 wurden 1516 stationäre und 366 tagesklinische Patienten behandelt. Zudem ist Dr. Martin Kaatz nach erfolgreicher Habilitation zum Privatdozenten ernannt worden, verbunden mit einer umfangreichen Lehr- und Vortragstätigkeit. Die Schwerpunkte der Klinik werden unter seiner Leitung kontinuierlich ausgebaut, darunter die Dermatoonkologie mit der Teilnahme an zahlreichen Therapiestudien sowie der anstehenden Zertifizierung als Hauttumorzentrum 2013/2014. Den Bereich der operativen Dermatologie vertritt seit dem Wechsel von Frau OÄ Durnick nach Berlin Frau OÄ Schell mit einem breiten Angebot dermatochirurgischer Leistungen wie z.B. der operativen Entfernung von Hauttumoren mit Verschiebelappenplastiken, Meshgraft mit anschließender Vakuumpumpentherapie, SentinelLymphknoten-Extirpation bei Melanompatienten, Elektrochemotherapie, großflächige Exzisionen bei Akne inversa oder kongenitalen Naevi bei Kindern. Auch die Analyse und Dokumentation melanozytärer Naevi von Patienten mit dysplastischem Naevuszellsyndrom mittels digitaler Dermatoskopie ist möglich. Die Diagnostik wird durch sonografische Untersuchungen der Lymphknoten, Haut und venösen Gefäße ergänzt ( $\bullet$ Abb.5). Im Bereich der konservativen Dermatologie konnte die extrakorporale Photopherese bei Patienten mit Kollagenosen, Lymphomerkrankungen, atopischer Dermatitis sowie GvHD etabliert werden. Weiterhin bietet die Klinik ein breites Spektrum an Lichttherapien, darunter UVA-1, PUVA, Sole-UVB-311 nm und ebenso die Möglichkeit einer PDT-Behandlung bei Feldkanzerisierung und aktinischen Keratosen. Umfangreiche allergologische Testungen bei Atopikern und Patienten mit Handekzemen mit berufsdermatologischer Fragestellung können ebenfalls erfolgen. Weiterhin ist die Durchführung der stationären Immuntherapie (SCIT) bei Insektengiftallergien, mit anschließender Stich-Provokationstestung, in Zusammenarbeit mit der Klinik für Anästhesiologie in Intubationsbereitschaft möglich. Patienten mit Lymphödemen/Lipolymphödemen erhalten, wenn notwendig, neben manueller und apparativer Lymphdrainage eine Anbindung an das Adipositaszentrum im Hause.

Weiterhin konnte unter Leitung von PD Dr. Martin Kaatz ein Studienzentrum aufgebaut werden, das nicht nur Studien im Bereich der Dermatologie betreut, sondern Projekte aus Wissenschaft und klinischer Forschung in allen Kliniken, Instituten und Fachbereichen des SRH-Waldklinikums unterstützt. Vom BMBF wird das Projekt „Woundoptomizer“ gefördert, in dem mit der Multiphotonenlasertomografie akute und chronische Wunden untersucht werden und das in Zusammenarbeit mit der Hautklinik des Universitätsklinikum Jena und Mannheim durchgeführt wird. Obwohl das „Zentrum für klinische Studien“ erst im April 2011 eröffnet worden ist, können wir bereits auf eine sehr erfolgreiche Zeit zurückblicken.

Im Jahr 2014 ist zudem der Umzug in den Neubau des Klinikums geplant. Gegenwärtig laufen intensive Vorbereitungen zur optimalen Konfiguration der Funktionsbereiche, der Diensträume und der Station.

Insgesamt hat die moderne Dermatologie in Deutschland mit ihren zahlreichen Teildisziplinen hervorragende Zukunftsaussichten und kann, wenn sie sich weiter als ein Fachgebiet mit breitem Diagnostik- und Therapieangebot betrachtet, optimistisch in die nächsten Jahre sehen. Wichtig dabei ist die enge Verzahnung zwischen stationärer und ambulanter Dermatologie unter intensiver Einbindung der niedergelassenen Fachkollegen.

\section{Interessenkonflikt}

$\nabla$

Der Autor gibt an, dass kein Interessenkonflikt besteht.

\section{Abstract}

\section{History of the Dermatological Department in Gera, Past, Present and Future $\nabla$}

The dermatological clinic in Gera has a traditional history of more than 60 years and is an essential component in the inpatient management of dermatological patients in East Thuringia. Throughout its entire existence the early introduction of innovative therapeutic procedures for the treatment of dermatoses and skin tumours has been introduced. The reputation of the clinic is due above all to the work of ChA Meyer who was its medical director for more than 30 years. At present the clinic comprises 30 inpatient and 5 day-patient beds, modern functional facilities with an emphasis on phototherapy and allergology. Concomitantly, the surgical services have been markedly expanded in the past few years as a component of specialty dermato-oncology. Next year the clinic will move into a modern new building. 